\title{
KELEA (Kinetic Energy Limiting Electrostatic Attraction) Offers an Alternative Explanation to Existing Concepts Regarding Wave-Particle Duality, Cold Fusion and Superconductivity
}

\author{
W. John Martin \\ Institute of Progressive Medicine, South Pasadena, USA \\ Email: wjohnmartin@ccid.org
}

How to cite this paper: Martin, W.J. (2016) KELEA (Kinetic Energy Limiting Electrostatic Attraction) Offers an Alternative Explanation to Existing Concepts Regarding Wave-Particle Duality, Cold Fusion and Superconductivity. Journal of Modern Physics, 7, 1995-2007.

http://dx.doi.org/10.4236/jmp.2016.715176

Received: October 7, 2016

Accepted: November 6, 2016

Published: November 9, 2016

Copyright $\odot 2016$ by author and Scientific Research Publishing Inc.

This work is licensed under the Creative Commons Attribution-NonCommercial International License (CC BY-NC 4.0).

http://creativecommons.org/licenses/by-nc/4.0/

\section{(c) (i) (8) Open Access}

\begin{abstract}
Existing explanations for several major phenomena in physics may need to be reconsidered in light of the description of a natural force termed KELEA (kinetic energy limiting electrostatic attraction). Three examples are selected for discussion in this paper: i) The proposed wave-particle duality of electrons; ii) cold fusion; and iii) superconductivity. The current interpretations of these enigmatic concepts are incomplete and not fully validated by scientific methods. The observations underlying these processes are seemingly consistent with KELEA acting as a repelling force between opposite electrical charges. Relatively simple experiments can be designed to either confirm or exclude KELEA in these and in various other currently perplexing physical phenomena.
\end{abstract}

\section{Keywords}

KELEA, Kinetic Energy Limiting Electrostatic Attraction, LENR, Low Energy Nuclear Reaction, Wave-Particle Duality, Double Slit Experiment, Cold Fusion, Deuterium, Palladium, Superconductivity, Condensed Matter Nuclear Science, Brown's Gas, Electrolysis, Activated Water

\section{Introduction}

A natural force termed KELEA (kinetic energy limiting electrostatic attraction) has been described in regards to the activation of water and other fluids [1] [2] [3] [4]. The proposed fundamental role of KELEA is to prevent the fusion and possible annihilation of electrostatically attracted opposite electrical charges. It may also explain the repulsion 
between positive and negative electrical charges. Within water, KELEA leads to a loosening of the hydrogen bonding of the water molecules [5]. These changes decrease the surface tension and increase the volatility of the water [6]. Moreover, KELEA activated water can enhance various biological activities, resulting in human, animal and agricultural benefits [7].

KELEA may also be related to various scientific phenomena. For example, environmental levels of KELEA may be subject to natural fluctuations, which may explain minor changes in measured weights of solid objects over time [8]. KELEA may also accompany cosmic rays and participate in the formation of cloud condensation nuclei (CCN) in helping to maintain the cloud barrier to solar global warming [9].

Quantum mechanics and other aspects of modern physics are predicated on the assumption that certain physical phenomena defy rational explanations in terms of intuitive, commonsense thinking. The concept of KELEA may help in reconciling some of the outstanding controversies, which are largely based on a belief system rather than formal proof. Such considerations may, in turn, shed further light into KELEA and its many potential applications within science and industry. This paper provides three examples of how the concept of KELEA may be relevant to modern physics.

\section{Wave-Particle Duality of Electrons as Evidenced by the Double Slit Experiment}

KELEA provides an alternative to wave-particle duality of electrons and other entities [10]. The argument that electrons have an intrinsic wave property is largely based on theoretical prediction [11] and actual observations [12] [13] made when a stream of individual electrons is transmitted through two adjacent slits onto a detecting screen. It is hypothesized that the individual electrons are traveling as a wave, which simultaneously enters the two slits and emerges as separate waves. The two emerging waves seemingly interact in a constructive and destructive interference pattern prior to contacting the detecting screen. Upon reaching the detecting screen the leading edge of the composite wave is thought to "collapse" to yield an electron particle. The explanation of the double slit experiment is further complicated by assertions to the effect that if the electrons are detected either as they pass through the slits or even after they have passed through the slits but before striking the detection screen, then the interference pattern is no longer observed. Instead the individual electrons are detected as if they passed through the slits as discrete particles and landed on the detecting screen directly aligning to the positions of the two slits.

The necessity of ascribing an intrinsic wave quality to electrons is obviated by assuming that KELEA comprises an actual wave function that can accompany electrons as they traverse space. In other words, rather than considering electrons as being both particles and waves, they may simply be particles accompanied by KELEA.

According to this scenario, the KELEA accompanying the electron extends as a field well beyond the physical confines of the electron. The wave-like function of KELEA would pass through both slits, while the individual electron particles would go through 
one or other of the two slits. The KELEA going through the non-electron traversing slit would be expected to re-associate with the KELEA remaining with the traversing electron, presumably in a fluctuating wave-like manner. Resulting regions of increased KELEA may exert a mutually attracting influence on the traversing electron, causing the electron to veer either towards or away from the center of the detecting screen. Such variable interactions of the electrons with KELEA coming from both slots could yield the overall multiple banding pattern of apparent constructive and destructive interference. Rather than attracting a waveform, it has also been proposed that particles could induce a waveform within its surrounding environment [14]. The example cited is that of ripples developing on water onto which a silicone drop is allowed to bounce. The ripples can be reflected from different surfaces and, thereby, provide a "pilot wave" that can affect the horizontal motion of the silicone drop [15].

The foundation of the double slit experiment was an early effort to prove that light comprises waves in addition to photons. Somewhat analogous to the "pilot wave" explanation, it could be argued that the fluctuating electrical and magnetic fields are responses to the moving photons. The fields are, therefore, locally induced reactions to the photons and not intrinsically parts of the photons. The induced fluctuating electrical fields could, however, attract KELEA. By converging multiple fluctuating lights to a central location, it was possible to increase the attraction of KELEA [16]. This principle, as well as a similar approach using bidirectional electrical currents, has been used in KELEA activation of water [17].

As noted above, electrons apparently behave as particles in the double slit experiment if they are monitored during their movement towards the detecting screen. The association of KELEA with the electron is reversible and can be at least partially withdrawn by competing electrical charges [9]. This could occur by the operation of the electronic equipment needed to determine the actual slit through which each individual electron is passing or has passed. Therefore, it is possible that efforts at determining the slit through which each electron had passed, are simply removing the influence of KELEA on the trajectories of the electrons.

In terms of providing fresh insight into KELEA, it is notable that interference patterns are also seen with protons [18], neutrons [19] and even whole molecules [20] [21] [22] [23]. KELEA attraction to protons is expected because of their positive electrical charge. For neutrons, however, it has to be assumed that positive and negative electrical charges of the quarks may also need to bind with KELEA to prevent electrical fusion [24]. With regards to more complex molecules, they possess ample electrical charges to attract KELEA. As discussed below, many dipolar molecules are particularly effective in attracting KELEA from the environment with its subsequent transfer to water.

\section{Cold Fusion}

The essence of the1989 report of "cold fusion" by Fleischmann and Pons is that electrolysis of deuterium water (heavy water) using palladium as the cathode and lithium deuteroxide as the electrolyte, leads after some time to transient periods (bursts) of 
greater heating of the water than attributable to the electrical input into the system [25]. The investigators further reported that low levels of tritium, neutrons and gamma rays were being released from the fluid. The formation of tritium was ascribed to nuclear fusion of deuterium atoms trapped (condensed) within the palladium cathode. Nuclear fusion would also explain the release of gamma rays. Deuterium fusion was further presumed to be leading to the production of helium; accounting for the proposed release of neutrons. The most striking conclusion, however, was that most of the deuterium fusion-derived energy was occurring as heat [26] [27]. The strong bias towards heat production rendered the supposed fusion process far more efficient and safer than the standard high temperature and pressure induced nuclear fusion in nuclear power plants [28]. Potentially the new process, termed "cold fusion," or more formally "Low Energy Nuclear Reaction (LENR)" and "Chemically Assisted Nuclear Reaction (CANR)" could provide much of the world's energy needs (www.lenr-canr.org).

The reports by Dr. Fleischmann and his colleagues withheld important details regarding methodology and reliability of the findings; presumably to help protect anticipated commercial benefits [29]. Yet the provocative although incomplete publications, stimulated many efforts to replicate and clarify the findings. Using similar systems, various investigators were able to confirm the excess heat occurring during long-term electrolysis of deuterium water using palladium as the cathode [30] [31] [32]. Detailed analyses have also included the additional energy output involved in the electrolysis dissociation of deuterium water to deuterium gas and oxygen. The majority of these investigators failed, however, to validate the presence of tritium, neutrons or gamma waves; questioning whether nuclear reactions were actually occurring [33] [34] [35]. The focus shifted to the detection by some investigators of helium, another indicator of likely nuclear fusion [36] [37] [38]. The standard method of helium analysis has been to pass the electrolysis gas through low temperature charcoal prior to spectroscopy. The charcoal is intended to remove oxygen and deuterium gas, but not helium. In reality, charcoal can weakly bind helium, which is commonly used in the spectroscopic laboratories. Dissociation of some charcoal-bound helium by the electrolysis gases could potentially explain the positive results from the cold fusion advocates [39] [40] [41]. It is also possible that small amounts of helium could have preexisted in the palladium electrode and simply be displaced by the deuterium gas [42] [43] [44].

Cold fusion research has steadily contradicted some of the earlier assumptions, while generally confirming the unexplained increased production of heat. Among the major developments is the finding that not all palladium electrodes are effective. Indeed, palladium is no longer regarded as being critical in the choice of electrodes since different metals, such as tin, nickel, titanium, gold, etc. can be used [45] [46]. Nor is deuterium critical since excess heat output over energy input can occur in electrolysis with regular (light) water [46] [47] [48]. The proposed concept has emerged that either hydrogen or deuterium gas is condensed via electrolysis within the lattice structures of certain metals and other materials, in a manner that can leads to atomic fusion. For certain compounds, such as zeolites, no electrolysis is needed since direct infusion with deuterium 
can lead to an increased temperature [49]. Similarly, even after the current to the electrodes in electrolysis is discontinued, the electrolysis fluid can continue to heat beyond the environmental temperature [50].

The primary argument that the excess heat being generated is derived by an intrinsic nuclear reaction is obviated by the concept of KELEA as an externally derived force. Many studies indicate an association of electrolysis with activation of water and, therefore, with KELEA becoming incorporated into the water [1] [2] [3] [4]. It is noteworthy that in addition to generating hydrogen gas at the cathode and oxygen gas at the anode, a third stream of vapor bubbles can commonly be observed during electrolysis of water. This steam of vapor bubbles emerges midway between the two electrodes, which is the interface between the opposite electrical charges emanating from the electrodes. The stream of vapor bubbles is likely to comprise KELEA activated water molecules, which subsequently become mixed with the oxygen and hydrogen gases coming from the anode and cathode, respectively.

Various industrial, agricultural and medical uses have been described for this water vapor gas, usually when used along with the electrolysis induced hydrogen and oxygen gases. For example, the use of the gas in welding was first described by Rhodes and Henes [51] and later shown by Yul Brown, for whom the gas is named [52] [53]. When ignited, Brown's gas has a temperature of approximately $135^{\circ} \mathrm{C}$ in air. Yet the flame can transfer high amounts of heat into various materials, including the ability to melt tungsten at $>3000^{\circ} \mathrm{C}$ [54]. Indeed, temperatures reaching nearly $6000^{\circ} \mathrm{C}$ have been achieved [55].

Brown's gas that is not ignited can be bubbled through regular water as a relatively easy method for attracting KELEA into water for human and animal consumption [56]. Pain relief from various medical conditions is achievable by simply applying Brown's gas over the affected areas [57].

Electrolysis of water can be achieved without any external energy supply by submerging two electrodes comprising materials with differing electrochemical potential into an electrolyte containing water solution and connecting the electrodes with a conducting wire. Alessandro Volta originally described this process, as well as defining the relative electrochemical potential of various metals [58]. His work led to the development of batteries, essentially by placing two dissimilar metals in contact with electrolytes, so as to allow electrical transmission between the metals. Using suitable resistance in the connecting wire, the electrical transmission can be rendered intermittent, essentially providing on-off switching. This simple procedure is comparable to more complicated electrical on-off switching devices, which have also been used to activate water. Examples include devices based on the beam ray of Royal Raymond Rife [59], the multiwave oscillator of Georges Lakhovsky [60], and the pulsed magnetic device of Panos Pappas [61].

Many dipolar compounds with separated electrical charges can directly absorb KELEA from the environment and transfer the energy to nearby water, possibly in an oscillatory manner. Prominent examples include zeolites, humic acids, mica, shungite 
(a fullerene product from Russia), ground volcanic rock, magnesium oxide and others [1] [2] [3]. It is not unreasonable to assume that even the electrons in a deuterium infused palladium electrode may occasionally create a highly efficient KELEA attracting antenna. Once water is sufficiently activated, its separated electrical charges can also attract further KELEA.

The transfer of kinetic energy to the water will result in a rise in temperature. This has been measured as increased infrared radiation emitted from water in which ground and heated volcanic rock pellets have been added. Since KELEA activated water has a lower specific heat compared to regular water, the same amount of energy input will increase the temperature of activated water beyond that of non-activated water [6]. Activated water can evaporate at a reduced temperature, further leading to increased estimates of the amounts of hydrogen and oxygen gas being formed during electrolysis. Finally, KELEA may also increase the ionization of the atmosphere surrounding the water. This could possibly influence the readings on certain electronic devices, such as scintillation counters.

KELEA can be attracted from the environment using various additional devices. Among the simplest are spaces enclosed within materials with alternating electrically conductive and insulating properties. Wilhelm Reich used such chambers to activate water upon the assumption that the chambers were capturing an external energy force that he termed Orgone [62]. He once demonstrated the increased ambient temperature within such a chamber to Albert Einstein [63], who seemingly failed to appreciate its significance [64].

Another emerging concept among cold fusion advocates is possible transmutation, that is, the induced conversion of an element to another element. Although not well documented, there have been suggestions of iron detection on gold cathodes [65] [66] and copper formation on nickel electrodes, during electrolysis in deuterium water. Not surprisingly, there have been similar claims for formation of various elements on electrodes used for the production of Brown's gas [54] [55]. The mineral identification is generally performed using Energy Dispersive X-Ray Spectra (EDX) analyses. EDX measures the energy levels of inner electrons between the $\mathrm{K}$ and $\mathrm{L}$ shells, which can be used as distinguishing characteristics of different minerals [67]. If KELEA can affect the strength of opposing electrical charges, it is conceivable that it may have an effect on the inner electron orbits, thereby, potentially yielding slightly anomalous results. This question arose in EDX studies on minerals within KELEA attracting particles, which form in certain virus infected cell cultures. EDX analyses clearly suggested the presence of titanium along with other elements that were never added to the cultures [68]. Whether it reflects true transmutation or an artifact of EDX caused by KELEA needs to be resolved. Studies with the flame of ignited Brown's gas have shown reduced measured radioactivity of ${ }^{60}$ cobalt [69]. This could either reflect transmutation or interference with the reliability of the measuring device.

Although not the primary topic of this paper, KELEA activated water shows clear evidence of abiotic chemical synthesis of lipid-related molecules [68]. 


\section{Superconductivity}

Electrons can be moved through certain materials in response to an applied positive electrical charge (voltage). The movement of electrons as particles can be impeded by the outstanding positive electrical charges of the protons of the atoms comprising the material. The moving electrons can also induce a minor magnetic field, acting in the reverse of the direction of the electrons. If electrons were waves, their movement could also be potentially impeded by vibrational waves (phonons) occurring within the material [70]. The availability and positioning of electrons, which can be moved through a material varies widely between different types of materials. The inverse relationship between the numbers of moving electrons (current) and the applied voltage is referred to as electrical resistance, which typically corresponds to the production of heat occurring within the material. The heat is attributed to the transduction of electrical energy to thermal energy due to friction of the electrons.

A complete absence of resistance to electrical currents, termed superconductivity [71] [72], can occur with some materials under certain circumstances. It was initially identified at temperatures barely above absolute zero, with an example being liquid helium maintained below $2.17^{\circ} \mathrm{K}$ [73]. More recently, materials are being identified that also show no electrical resistance, but up to far higher temperatures, although still considerably below room temperature. By definition, there is no electron friction and essentially no magnetic field generated in these "high temperature" superconductors. It is as if below a critical temperature, the moving electrons abruptly disengage from the positive electrostatic charges of the protons. One suggestion for explaining this phenomenon is that the electrostatic attraction is nullified when electrons engage in pair formations. The paired electrons are thought to remain physically separated, yet they still apparently need to overcome the normal electrical repulsion between negatively charged particles. For very low temperature superconductivity and possibly some examples of high temperature superconductivity, the paired electrons (Cooper pairs) may become coupled by phonons oscillating between the two electrons [74]. It is proposed that the positive charges within the conducting material are unable to distort the pair formation if interactive with only one of the electrons comprising each set of paired electrons.

Recent consideration is being given to other potential electron coupling mechanisms in high temperature superconductors. One suggestion is reciprocating electron spin [75]. The pairing may also be occurring between high-energy outer shell electrons (heavy electrons) [76] [77] or between electrons with reduced mass and/or electrical charges (majorana fermions) [78]. It seems that the efficiency of higher temperature superconductivity correlates with increasing numbers of electron pairs and with smaller sized pairs.

A more straightforward suggestion is that KELEA directly reduces electrical resistance by simply voiding the electrostatic attractions between electrical-driven electrons and the positive charges within the conducting materials. KELEA could also help compel the movements of electrons by adding to their mutually repulsive force. Consistent 
with the involvement of KELEA in electrical transmission, there are anecdotal observations suggesting that household electrical bills can be reduced by strategically placing sets of magnets on both electrical and plumbing lines. If true, the magnets could possibly be directing an inflow of KELEA into the electrical line, thereby creating less resistance.

There is a convergence between the types of complex molecules being utilized in superconducting research and compounds being suggested for use as cathodes in cold fusion reactions. While some of these compounds are naturally occurring, others are being either completely synthesized or are unique combinations of elements not normally found together in Nature. Some of this research comes under the heading of Condensed Matter Nuclear Science (CMNS). Examples of compounds, which feature in both superconductivity and cold fusion research are zeolites (aluminosilicates), especially if doped with additional metallic species [79]; perovskite organometal structures [80], such as methyl ammonium lead iodide; cuprates; fullerenes; clathrates; dendrimers; and aromatic hydrocarbons [81] [82] [83] [84] [85]. Prior heating in non-oxidizing environments can commonly enhance their subsequent reactivities when tested under standardized conditions.

Similar groupings of products are being studied for their opticoelectronics properties, such as uses in electrical transmission within solar panels [86]. These products have marked dipolar characteristics, criteria that can render a material effective as an antenna able to attract KELEA. It will be surprising if KELEA is not playing a major role in the physics of "condensed matter".

\section{Conclusions}

Scientific progress commonly follows the formulation of an alternative explanation, which challenges an accepted and seemingly proven scientific belief. If sufficiently plausible, the newly offered explanation reduces the standing of the previously accepted belief to that of being plausible but not proven. Experiments can then be conducted to differentiate between the two competing alternatives. If the experiments disprove one of the alternatives, the surviving explanation is likely to gain in status to an accepted belief. This designation is, nevertheless, only provisional and removed if a new alternative, plausible explanation emerges.

The increasing electrostatic attraction of approaching opposite electrical charges would seemingly lead to the fusion and possible annihilation of the charges were it not for the intervention of a countervailing force. In the case of an electron directly approaching a proton, it clearly enters into an orbit, yielding a hydrogen atom. This change in electron trajectory requires kinetic force acting upon the electron. The concept of kinetic energy was actually developed not from this consideration, but from the movement patterns of dissolving and non-dissolved particles of neutral red dye suspended in activated water. Unlike the slowly expanding circular spread of dissolving dye in non-activated water, the dye particles proceeded in a to-and-fro movement leading to long streaks of dye. Moreover, in clusters of non-dissolved particles, individual particles would be periodically rejected from the cluster. The particles would slowly 
return towards the cluster only to be repelled once again. Many additional observations have since been recorded that are consistent with the existence of KELEA.

Sufficient information is, therefore, available regarding KELEA to design meaningful experiments. These experiments can include testing whether KELEA can i) affect the trajectory of electrons; ii) raise the temperature of fluids; and iii) diminish electrical resistance. The testing can then be directly applied to the systems on which the conventional concepts are based. Thus, KELEA can be imposed onto the two-slit experiment, electrolysis of deuterium water and an electrical circuit. If effects do occur, they may become adapted to useful quantitative assays for KELEA.

The three examples chosen for discussion in this paper can be extended to many additional possible involvements of KELEA in other enigmatic physical phenomena. Examples of such analyses will be provided in subsequent publications.

\section{Acknowledgements}

The Institute of Progressive Medicine is a component of MI Hope Inc., a non-profit public charity.

\section{References}

[1] Martin, W.J. (2014) Stealth Adapted Viruses; Alternative Cellular Energy (ACE) \& KELEA Activated Water. Author House, Bloomington, IN, p. 321.

[2] Martin, W.J. (2015) International Journal of Complementary \& Alternative Medicine, 1, 00001. http://dx.doi.org/10.15406/ijcam.2015.01.00001

[3] Martin, W.J. (2015) International Journal of Complementary \& Alternative Medicine, 2, 00051. http://dx.doi.org/10.15406/ijcam.2015.02.00051

[4] Martin, W.J. (2016) Journal of Transportation Technologies, 6, 148-154. http://dx.doi.org/10.4236/jtts.2016.63014

[5] Martin, W.J. (2015) Open Journal of Biophysics, 5, 69-79. http://dx.doi.org/10.4236/ojbiphy.2015.53006

[6] Martin, W.J. (2015) Open Journal of Energy Efficiency, 4, 36-43. http://dx.doi.org/10.4236/ojee.2015.42004

[7] Martin, W.J. (2014) Advances in Plants \& Agriculture Research, 2, 00033.

[8] Martin, W.J. (2016) Journal of Modern Physics, 7, 461-472. http://dx.doi.org/10.4236/jmp.2016.76048

[9] Martin, W.J. (2016) Atmospheric and Climate Sciences, 6, 174-179. http://dx.doi.org/10.4236/acs.2016.62015

[10] Selleri, F. (2012) Wave Particle Duality. Springer, Berlin, p. 206.

[11] Feynman, R., Leighton, R.B. and Sands, M.L. (1965) The Feynman Lectures on Physics: Quantum Mechanics. Vol. 3, Chapter 1, Addison-Wesley, Reading, MA.

[12] Frabboni, S., Gabrielli, A., Gazzadi, G.C., Giorgi, F., Matteucci, G., Pozzi, G., Cesari, N.S., Villa, M. and Zoccoli, A. (2012) Ultramicroscopy, 116, 73-76. http://dx.doi.org/10.1016/j.ultramic.2012.03.017

[13] Bach, R., Pope, D., Liou, S.-H. and Batelaan, H. (2013) New Journal of Physics, 15, Article ID: 033018. http://dx.doi.org/10.1088/1367-2630/15/3/033018 
[14] Bohm, D. (1952) Physical Review, 85, 180-193. http://dx.doi.org/10.1103/PhysRev.85.180

[15] Mahler, D.H., Rozema, L., Fisher, K., Vermeyden, L., Resch, K.J., Wiseman, H.M. and Steinberg, A. (2016) Science Advances, 2, e1501466. http://dx.doi.org/10.1126/science.1501466

[16] Martin, W.J. (2015) Open Journal of Biophysics, 5, 115-121. http://dx.doi.org/10.4236/ojbiphy.2015.54010

[17] Martin, W.J. (2015) International Journal of Complementary \& Alternative Medicine, 1, Article ID: 00034. http://dx.doi.org/10.15406/ijcam.2015.01.00034

[18] Chadderton, L.T. (1996) Physics Letters, 23, 303-304. http://dx.doi.org/10.1016/0031-9163(66)90009-6

[19] Zeilinger, A., Gähler, R., Shull, C.G., Treimer, W. and Mampe, W. (1988) Reviews of Modern Physics, 60, 1067-1073. http://dx.doi.org/10.1103/RevModPhys.60.1067

[20] Carnal, O. and Mlynek, J. (1991) Physical Review Letters, 66, 2689-2692. http://dx.doi.org/10.1103/PhysRevLett.66.2689

[21] Arndt, M., Nairz, O., Vos-Andreae, J., Keller, C., van der Zouw, G. and Zeilinger, A. (1999) Nature, 401, 680-682. http://dx.doi.org/10.1038/44348

[22] Eibenberger, S., Gerlich, S., Arndt, M., Mayor, M. and Tüxen, J. (2013) Physical Chemistry Chemical Physics, 15, 14696-14700. http://dx.doi.org/10.1039/c3cp51500a

[23] Gerlich, S., Eibenberger, S., Tomandl, M., Nimmrichter, S., Hornberger, K., Fagan, P.J., Tüxen, J., Mayor, M. and Arndt, M. (2011) Nature Communications, 2, Article No. 263. http://dx.doi.org/10.1038/ncomms1263

[24] Gudder, S.P. (1984) Wave-Particle Duality in a Quark Model. In: Diner, S., Fargue, D., Lochak, G. and Selleri, F., Eds., The Wave-Particle Dualism, Reidel Publishing Company, Boston, 499-514. http://dx.doi.org/10.1007/978-94-009-6286-6 28

[25] Fleischmann, M. and Pons, S. (1989) Journal of Electroanalytical Chemistry, 261, 301-308 and errata in 263, 187-188.

[26] Fleischmann, M., Pons, S., Anderson, M.W., Li, L.J. and Hawkins, M. (1990) Journal of Electroanalytical Chemistry and Interfacial Electrochemistry, 287, 293-348. http://dx.doi.org/10.1016/0022-0728(90)80009-U

[27] Pons, S. and Fleischmann, M. (1990) Fusion Science and Technology, 17, 669-679.

[28] Olsson, P. and Aasen, A. (2009) Nuclear Reactors, Nuclear Fusion and Fusion Engineering. Nova Science Publishers, New York, 484.

[29] Peat, F.D. (1989) Cold Fusion: The Making of a Scientific Controversy. Contemporary Books, Chicago, 188.

[30] Iwamura, Y., Itoh, T., Gotoh, N. and Toyoda, I. (1998) Fusion Science and Technology, 33, 476-492.

[31] Liaw, B.Y., Tao, P.-L., Turner, P. and Liebert, B.E. (1991) Journal of Electroanalytical Chemistry and Interfacial Electrochemistry, 319, 161-175. http://dx.doi.org/10.1016/0022-0728(91)87075-F

[32] Storms, E.K. (1993) Fusion Science and Technology, 23, 230-245.

[33] Salamon, M.H., Wrenn, M.E., Bergson, H.E., Crawford, H.C., Delaney, W.H., Henderson, L., et al. (1990) Nature, 344, 401-405. http://dx.doi.org/10.1038/344401a0

[34] Bertin, A., Bruski, M. and Bystritskij, V.M. (1994) Yadernaya Fizika (Russian), 59, 976-980.

[35] Huizenga, J.R. (1992) Cold Fusion: The Scientific Fiasco of the Century. Oxford University Press, Oxford, 318. 
[36] Bush, B.F., Lagowski, J.J., Miles, M.H. and Ostrom, G.S. (1991) Journal of Electroanalytical Chemistry and Interfacial Electrochemistry, 304, 271-278. http://dx.doi.org/10.1016/0022-0728(91)85510-V

[37] Miles, M.H., Hollins, R.A., Bush, B.F., Lagowski, J.J. and Miles, R.E. (1993) Journal of Electroanalytical Chemistry, 346, 99-117. http://dx.doi.org/10.1016/0022-0728(93)85006-3

[38] Miles, M.H. and Bush, B.F. (1994) Transactions of Fusion Technology, 26, 156-159.

[39] McLean, S. (1918) Transaction of the Royal Society of Canada Series III, 12, 79-81.

[40] Dacey, J.R. and Edwards, M.H. (1964) Canadian Journal of Physics, 42, 241-250. http://dx.doi.org/10.1139/p64-024

[41] Duband, L., Ravex, A. and Chaussy, J. (1987) Cryogenics, 27, 397-400. http://dx.doi.org/10.1016/0011-2275(87)90211-6

[42] Abell, G.C., Matson, L.K., Steinmeyer, R.H., Bowman Jr., R.C. and Oliver, B.M. (1990) Physical Review B, 41, 1220. http://dx.doi.org/10.1103/PhysRevB.41.1220

[43] Camp, W.J. (1977) Journal of Vacuum Science \& Technology, 14, 514-517. http://dx.doi.org/10.1116/1.569292

[44] Jung, P. (1991) Diffusion and Clustering of Helium in Noble Metals. In: Donnelly, S.E. and Evans, J.H., Eds., Fundamental Aspects of Inert Gases in Solids, Plenum Press, New York, 59-66. http://dx.doi.org/10.1007/978-1-4899-3680-6 5

[45] Zhang, Q.F., Gou, Q.Q. and Zhu, H. (1995) Chinese Journal of Atomic and Molecular Physics, 12, 165-169.

[46] Rossi, A. (2015) Energy-Producing Reaction Devices, Systems and Related Methods. United States Patent Application Number 20160051957.

[47] Notoya, R. (1993) Fusion Science and Technology, 24, 202-204.

[48] Ohmori, T., Enyo, M., Mizuno, T., Nodasaka. Y. and Minagawa, H. (1997) Fusion Science and Technology, 31, 210-218.

[49] Sendis, A. (2011) Desktop Fusion of $\mathrm{D}_{2}$ in Palladium Nano-Particles Loaded in Zeolites. Senior Thesis Chemistry Department, University of La Verne May 25, 2011. Cited in Biberian, J.-P. and Parachamazad, Miles, M.H. (2014) The Journal of Condensed Matter Nuclear Science, 13, 38-43.

[50] Pons, S. and Fleischmann, M. (1994) Transactions of Fusion Technology, 26, 87-93.

[51] Rhodes, W. and Henes, R.A. (1962) Apparatus for the Electrolytic Production of Hydrogen and Oxygen for the Safe Combustion Thereof. United States Patent Number 3262872 Issued 26 July 1966.

[52] Brown, Y. (1974) Welding. US Patent Number 4,014,777 Issued on 29 March 1977.

[53] Brown, Y. (1976) Arc-Assisted Oxy/Hydrogen Welding. US Patent Number 4,081,656 Issued on 28 March 1978.

[54] Hurtak, J.J. and Hurtak, D. (2014) The History and Future of Brown's Gas. Nexus Magazine, June-July 2014, 49-54.

[55] Wiseman, G. (2009) Brown's Gas Book 2. Published by Eagle Research, Oroville, WA, 59.

[56] Martin, W.J. (2011) Methods for Increasing the Kinetic Activity of Alcohol, Water and Other Liquids, So as to Render the Liquids More Useful in Enhancing the Alternative Cellular Energy Pathway in the Prevention and Therapy of Diseases. United States Patent Application Number 20120207850.

[57] Kang, S.D. (2007) Use of Brown's Gas and Feeding Apparatus of the Brown's Gas. United States Patent Application Number 20070104797. 
[58] Bagotsky, V.S. (2005) Fundamentals of Electrochemistry. 2nd Edition, John Wiley \& Sons, Hoboken, 640. http://dx.doi.org/10.1002/047174199X

[59] Silver, N. (2001) The Handbook of Rife Frequency Healing: Holistic Technology for Cancer and Other Diseases. The Center for Frequency Education, CA, 430. $\square$

[60] Lakhovsky, G. (1996) The Secret of Life. Cosmic Rays and Radiations of Living Beings (Translated by Clement, M.). Health Research, Pomeroy, WA, 213.

[61] Pappas, P.T. and Wallach, C. (1993) Effects of Pulsed Magnetic Field Oscillations in Cancer Therapy. International Association for New Science, Fort Collins, CO.

[62] Reich, W. (1948) The Cancer Biopathy. The Discovery of the Orgone. Vol. 2 (Translated by White, A., Higgins, M. and Raphael, C.M. (1973)), Farrar, Straus and Giroux, New York, 433.

[63] Reich, W. (1953) The Einstein Affair. Orgone Institute Press, Ashland, OR.

[64] Correa, P.N. and Correa, A.N. (2010) Journal of Aetherometric Research, 2, 1-24.

[65] Ohmori, T., Mizuno, T., Minagawa, H. and Enyo, M. (1997) International Journal of Hydrogen Energy, 22, 459-463. http://dx.doi.org/10.1016/S0360-3199(96)00126-7

[66] Ohmori, T., Mizuno, T., Nodasaka, Y. and Enyo, M. (1998) Fusion Science and Technology, 33, 367-382.

[67] Brillson, L.J. (2016) An Essential Guide to Electronic Material Surfaces and Interfaces. John Wiley \& Sons, Hoboken, 320. http://dx.doi.org/10.1002/9781119027140

[68] Martin, W.J. (2003) Experimental and Molecular Pathology, 74, 210-223. http://dx.doi.org/10.1016/S0014-4800(03)00037-6

[69] Haley, D. (1993) Planetary Association for Clean Energy. Newsletter, 6, 8-9.

[70] Harris, R. (1999) Nonclassical Physics. Beyond Newton's View. Addison-Wesley, Menlo Park, 608.

[71] Bardeen, J., Cooper, L.N. and Schrieffer, J.R. (1957) Physical Review, 108, 1175. http://dx.doi.org/10.1103/PhysRev.108.1175

[72] Poole Jr., C.P., Farach, H.A., Creswick, R.J. and Prozorov, R. (2014) Superconductivity. 3rd Edition, Elsevier, Amsterdam, 870.

[73] Van Sciver, S.W. (2012) Helium Cryogenics. Springer Science, New York, 470. http://dx.doi.org/10.1007/978-1-4419-9979-5

[74] Combescot, M. and Shiau, S.-Y. (2016) Excitons and Cooper Pairs: Two Composite Bosons in Many-Body Physics. Oxford University Press, Oxford, 504.

[75] Zhao, J., Niestemski, F.C., Kunwar, S., Li, S., Steffens, P., Hiess, A., Kang, H.J., Wilson, S.D., Wang, Z., Dai, P. and Madhavan, V. (2011) Nature Physics, 7, 719-724. http://dx.doi.org/10.1038/nphys2006

[76] Pfleiderer, C. (2009) Reviews of Modern Physics, 81, 1551-1624. http://dx.doi.org/10.1103/RevModPhys.81.1551

[77] Aynajian, P., da Silva Neto, E.H., Gyenis, A., Baumbach, R.E., Thompson, J.D., Fisk, Z., Bauer, E.D. and Yazdani, A. (2012) Nature, 486, 201-206. http://dx.doi.org/10.1038/nature11204

[78] Lu, Y.-M. and Wang, Z.Q. (2013) Physical Review Letters, 110, Article ID: 096403. http://dx.doi.org/10.1103/physrevlett.110.096403

[79] Goldstein, T.P. (1967) Superconducting Molecular Sieves. United States Patent 3509071A.

[80] Engler, E.M., Lee, Y.Y., Nazzal, A.I., Beyers, R.B., Lim, G., Grant, P.M., et al. (1987) Journal of the American Chemical Society, 109, 2848-2849. http://dx.doi.org/10.1021/ja00243a061 
[81] Hirsch, J.E., Maple, M.B. and Marsiglio, F. (2015) Physica C: Superconductivity and Its Applications, 514, 1-8. http://dx.doi.org/10.1016/j.physc.2015.03.002

[82] Božović, I., He, X., Wu, J. and Bollinger, A.T. (2016) Nature, 536, 309-311. http://dx.doi.org/10.1038/nature19061

[83] Van Tendeloo, G., Antipov, E.V. and Putilin, S.N., Eds. (1999) High-Temperature Superconductors and Novel Inorganic Materials. In: Nato Science Partnership Subseries 3: Vol. 63, Springer, New York, 307.

[84] Takabayashi, Y. and Prassides, K. (2016) Philosophical Transactions of the Royal Society A: Mathematical, Physical and Engineering Sciences, 374, Article ID: 20150320. http://dx.doi.org/10.1098/rsta.2015.0320

[85] Kubozono, Y., Goto, H., Jabuchi, T., Yokoya, T., Kambe, T., Sakai, Y., et al. (2015) Physica C: Superconductivity and Its Applications, 514, 199-205. http://dx.doi.org/10.1016/j.physc.2015.02.015

[86] Solymar, L., Walsh, D. and Syms, R.R.A. (2014) Electrical Properties of Materials. Oxford University Press, Oxford, 484.

\section{Abbreviations}

KELEA: kinetic energy limiting electrostatic attraction; LENR: low energy nuclear reaction.

Submit or recommend next manuscript to SCIRP and we will provide best service for you:

Accepting pre-submission inquiries through Email, Facebook, LinkedIn, Twitter, etc.

A wide selection of journals (inclusive of 9 subjects, more than 200 journals)

Providing 24-hour high-quality service

User-friendly online submission system

Fair and swift peer-review system

Efficient typesetting and proofreading procedure

Display of the result of downloads and visits, as well as the number of cited articles

Maximum dissemination of your research work

Submit your manuscript at: http://papersubmission.scirp.org/

Or contact jmp@scirp.org 